\title{
LabonaChip
}

\section{Microfluidics-based single cell analysis reveals drug- dependent motility changes in trypanosomes}

\begin{tabular}{|r|l|}
\hline Journal: & Lab on a Chip \\
\hline Manuscript ID: & LC-ART-02-2015-000124 \\
\hline Article Type: & Paper \\
\hline Date Submitted by the Author: & 02-Feb-2015 \\
\hline Complete List of Authors: & $\begin{array}{l}\text { Hochstetter, Axel; University of Basel, Department of Chemistry } \\
\text { Stellamanns, Eric; Max Planck Institute for Dynamics and Self- } \\
\text { Organization, Department of Complex Fluids } \\
\text { Deshpande, Siddharth; University of Basel, Department of Chemistry } \\
\text { Uppaluri, Sravanti; Max Planck Institute for Dynamics and Self- } \\
\text { Organization, Department of Complex Fluids } \\
\text { Engstler, Markus; University of Würzburg, Department of Cell and } \\
\text { Developmental Biology, Biocentre } \\
\text { Pfohl, Thomas; University of Basel, Departement Chemie; Max Planck } \\
\text { Institute for Dynamics and Self-Organization, Department of Complex } \\
\text { Fluids }\end{array}$ \\
\hline
\end{tabular}




\section{Graphical Abstract}

Combination of microfluidics with optical tweezers is used in order to analyze the impact of drugs and chemicals on the motility of unicellular parasites

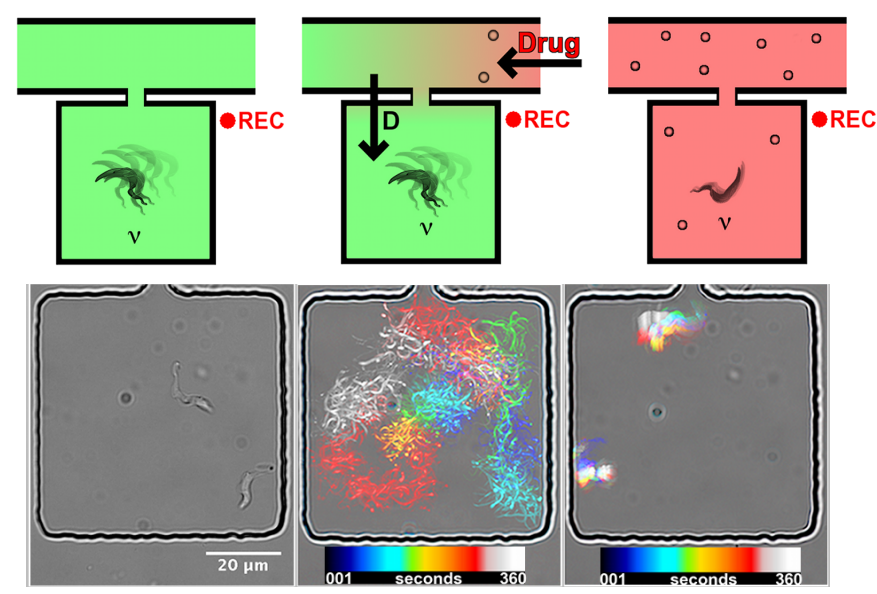




\title{
Lab on a Chip
}

\section{ARTICLE}

Cite this: DOI: 10.1039/x0xx00000x

\section{Microfluidics-based single cell analysis reveals drug- dependent motility changes in trypanosomes}

\author{
Axel Hochstetter ${ }^{a}$, Eric Stellamanns ${ }^{b, 1}$, Siddharth Deshpande ${ }^{a, 2}$, Sravanti \\ Uppaluri ${ }^{b, 3}$, Markus Engstler ${ }^{c}$, and Thomas Pfohl ${ }^{a, b, 4}$
}

Received 00th January 2012 Accepted 00th January 2012

DOI: $10.1039 / \times 0 \times x 00000 x$

www.rsc.org/

\begin{abstract}
We present a single cell viability assay, based on chemical gradient microfluidics in combination with optical micromanipulation. Here, we used this combination to in situ monitor the effects of drugs and chemicals on the motility of the flagellated unicellular parasite Trypanosoma brucei; specifically, the local cell velocity and the mean squared displacement (MSD) of the cell trajectories. With our method, we are able to record in situ cell fixation by glutaraldehyde, and to quantify the critical concentration of 2deoxy- $D$-glucose required to completely paralyze trypanosomes. In addition, we detected and quantified the impact on cell propulsion and energy generation at much lower 2-deoxy-D-glucose concentrations. Our microfluidics-based approach advances fast cell-based drug testing in a way that allows us to distinguish cytocidal from cytostatic drug effects, screen effective dosages, and investigate the impact on cell motility for drugs and chemicals. Using suramin, we could reveal the impact of the widely used drug on trypanosomes: Suramin lowers trypanosome motility and induces cell-lysis after endocytosis.
\end{abstract}

\section{Introduction}

The discovery of drugs that are effective in the battle against rapidly adapting pathogens requires concerted efforts with respect to developing improved screening assays and understanding of the mode of action of drugs on the target cell. To economize these efforts, pharmaceutical research increasingly employs cell culture assays that bridge the gap between molecular and animal drug testing. ${ }^{1}$ In many instances, analysis of whole cells (cellomics) ${ }^{2}$ allows for more accurate predictions of drug responses than molecular assays because the complex molecular interactions and the cellular compartments that significantly influence the cell's reaction to a drug are retained. ${ }^{1}$ Cell culture-based drug discovery commonly involves cell chips, high throughput screenings, and cell viability assays using fluorescence or luminescent transgenic target organisms. ${ }^{1,3-5}$ Often, these assay systems are unable to distinguish between drugs that kill the target cells (cytocidals) and drugs that merely inhibit cell growth (cytostatics). ${ }^{6}$

In recent years, microfluidics has emerged as a powerful tool for the discovery of drugs, as well as for elucidating their effects on cells, cell secretions, and signaling pathways. ${ }^{1,7-9}$ Moreover, microfluidics has proven useful for exploring individual cells in a heterogeneous population. ${ }^{7}$ The range of applications in single cell analysis is increased manifold by combining microfluidics with optical tweezers that allow the manipulation of cells by miniscule forces without touching. ${ }^{10,11}$ Examples include the sorting of motile cells ${ }^{12}$ and the characterization of cell motility ${ }^{13}$, but also specific placing of cells into defined environments. ${ }^{12,14,15}$

In single cell assays, the effects of environmental parameters are frequently assessed through analysing cell lysates. ${ }^{7,16,17}$ However, this type of endpoint analysis excludes further investigations of downstream effects. On the other hand, physical or biophysical properties of living cells so far are very rarely used as readout. The few published examples include monitoring bioelectrical impedance ${ }^{18}$ in response to drug treatment or migratory behavior. ${ }^{9}$ So far, the impacts of drugs onto highly motile unicellular pathogens have rarely been studied in single cell assays. ${ }^{2}$ The motility of non-selfproliferating spermatozoa however has been investigated on the single cell level with optical tweezers. ${ }^{19}$

Trypanosoma brucei, a unicellular organism belonging to the genus of trypanosomes, is one of the most rapidly adapting pathogens. ${ }^{20-24}$ The subspecies T. brucei brucei is endemic in sub-Saharan Africa where it causes Nagana disease in livestock $^{25}$, whereas $T$. brucei rhodesiense and T. brucei gambiense are the causative agents of the fatal sleeping 
sickness in humans (Human African Trypanosomiasis, HAT). ${ }^{26-30}$

Trypanosomes are extensively studied model flagellates. ${ }^{23,31,32}$ The flagellum is structurally different from bacterial flagella and more complex than most other eukaryotic flagella. ${ }^{33}$ Nonetheless, it is essential for locomotion and viability. Moreover, the flagellum is key to evading the immune response of the infected mammalian host. ${ }^{32}$

Present screening procedures for trypanocidal compounds involve either in vitro assays that take several hours ${ }^{5,6,34}$ or in vivo experiments that are based on establishing mutant trypanosomes that take weeks ${ }^{35}$ and therefore, are even more time-consuming.

Here, we introduce a real-time motility analysis system that is based on a straightforward microfluidic device in combination with time-resolved microscopy and optical tweezers. In the device, the concentration of drugs and chemicals is diffusioncontrolled and can be precisely tuned, with simultaneous observation of single cells. This setup allows us to physically characterize and quantify how drugs and chemicals affect trypanosome motility. Specifically, we quantify drug-induced reversible paralysis and irreversible chemical fixation of trypanosomes in a dose-dependent manner.

\section{Materials and Methods}

\section{Cell culture and solutions}

Trypanosoma brucei brucei MiTat 1.2 bloodstream form trypanosomes were routinely grown in HMI-9 ${ }^{36}$ culture medium at $37{ }^{\circ} \mathrm{C}$ in a $95 \%$ air $/ 5 \% \mathrm{CO}_{2}$ humidified atmosphere. Populations were kept below $10^{6}$ cells per $\mathrm{mL}$ by repetitive splitting. After a maximum of 15 splittings, trypanosomes were discarded and fresh populations were defrosted.

For experiments, $2 \mathrm{~mL}$ of trypanosomes in culture medium were centrifuged at $237 \mathrm{xg}$ and washed once with $2 \mathrm{~mL}$ of culture medium and once with $2 \mathrm{~mL}$ culture medium with bovine serum albumin (BSA, $5 \mathrm{mg} / \mathrm{mL}$ ). The supernatant was discarded and the cells were taken up into a $1 \mathrm{~mL}$ syringe (Braun) in $0.7 \mathrm{~mL}$ of culture medium with BSA $(5 \mathrm{mg} / \mathrm{mL})$. This comprised the cell solution, which was introduced into the device. The solution of culture medium and BSA was weekly prepared and stored at $37^{\circ} \mathrm{C}$ and at $5 \% \mathrm{CO}_{2}$ in humid atmosphere to ascertain optimal conditions.

For drug solutions of suramin, the drug was dissolved in $2 \mathrm{~mL}$ of culture medium and polystyrene beads $(1 \mu \mathrm{m}$ diameter, Polysciences) solution $2 \mu \mathrm{L} / \mathrm{mL}$. For drug solutions of GA and 2DG, $2 \mathrm{~mL}$ of culture medium solvent contained additionally BSA $(5 \mathrm{mg} / \mathrm{mL})$ and polystyrene beads $(1 \mu \mathrm{m}$ diameter, Polysciences) solution $2 \mu \mathrm{L} / \mathrm{mL}$.

\section{Device preparation}

The devices were produced using standard soft lithographic methods $^{37}$ and were made from PDMS (Dow Corning) covalently bound to glass cover slides ( $5 \mathrm{~cm}$ diameter, VWR). For the inlets and outlets, holes were punched out of the PDMS using a punching tool (0.5 $\mathrm{mm}$ diameter, Harris) into which PTFE-tubings (Fischer-Scientific) were inserted.

The syringes were equipped with cannulas $(0.45 \mathrm{~mm}$ diameter, Terumo), which were inserted tightly into the PTFE tubings and thus connected to computer-controlled neMESYS syringe pumps (Cetoni).

\section{Optical tweezers microscope and image analysis}

The optical tweezers setup consisted of a collimated diode laser $(\lambda=808 \mathrm{~nm}, \quad$ Schaefter + Kirchhoff $\mathrm{GmbH})$ with Gaussian beam-profile coupled into the light path of the microscope to generate a single beam gradient-trap. Images were recorded at one frame per second on an upright BX61 microscope (Olympus), equipped with a 40x water immersion objective $(\mathrm{NA}=1.15$, Olympus) and a Sensicam CCD-camera (PCO). The motion of trypanosomes before, during and after exposure to drugs was recorded at about 10 frames per second. The recorded image stacks were aligned using StackReg ${ }^{38}$ for Fiji ${ }^{39}$ in translation mode. Trajectories of moving trypanosomes within stacks were recorded using manual tracking for Fiji ${ }^{39}$. The obtained trajectories were analysed with MATLAB to retrieve the mean square displacements (MSDs) and the velocity plots of the recorded trajectories.

\section{Fluorescence Imaging}

Fluorescence imaging was conducted with an upright BX61 microscope (Olympus) and a Sensicam CCD-camera (PCO), using a xenon lamp, filter set $\left(\lambda_{E x c}=535 \mathrm{~nm}, A H F\right)$ and rhodamine B as fluorescent dye. Devices were first flushed with culture medium and BSA $(5 \mathrm{mg} / \mathrm{ml})$ from the cell inlet through the device, while the drug inlet was blocked (step I). Then, the inlet and the outlet on the cell side were blocked and fluorescent drug solution ran through drug side (step III) before the device was flushed with rhodamine B solution from the drug inlet as shown in figure $1 \mathrm{a}$, step IV. Time-count started when the dye-solution pushed out the cell media through the device. The fluorescence images (figure S1) were corrected for uneven illumination with Gaussian-like distribution.

For the experiments, only square chambers with a side length of $62.5 \mu \mathrm{m}$ and $75 \mu \mathrm{m}$ respectively were used, which explains the plateaus in the MSDs around 3900-5625 $\mu^{2}$. In smaller chambers, the trypanosomes would be too confined for proper MSDs while bigger chambers would be too big for the field of view of the camera.

\section{CFD simulations}

For the computational fluid dynamics (CFD) simulations we used the CFD module from the software COMSOL 4.3a. The microfluidic device was modeled in $3 \mathrm{D}$ using 54,167 finite 
elements. At first, the stationary laminar flow was calculated, whereas in a second step the time dependent material transport of the diluted species were calculated by using the beforehandobtained stationary flow fields.

CFD simulations of the distribution of a drug with a diffusion coefficient of $D_{\text {drug }}=5.7 .10^{-10} \mathrm{~m}^{2} / \mathrm{s}$ (representative for small molecules) are shown in supplementary video S1 and S2 and figure $\mathrm{S} 1$.

\section{Results}

\section{Design and function of the drug testing device}

Our experimental setup for single cell analysis combines microfluidics with time-resolved microscopy and optical tweezers. The microfluidic structure consists of one main channel (width: $40 \mu \mathrm{m}$, height: $9 \mu \mathrm{m}$ ), which is linked to adjacent square microchambers by connecting channels (width: $4 \mu \mathrm{m}$, length: $12 \mu \mathrm{m}$ ) and ends on both sides in a Y-fork (figure 1a).

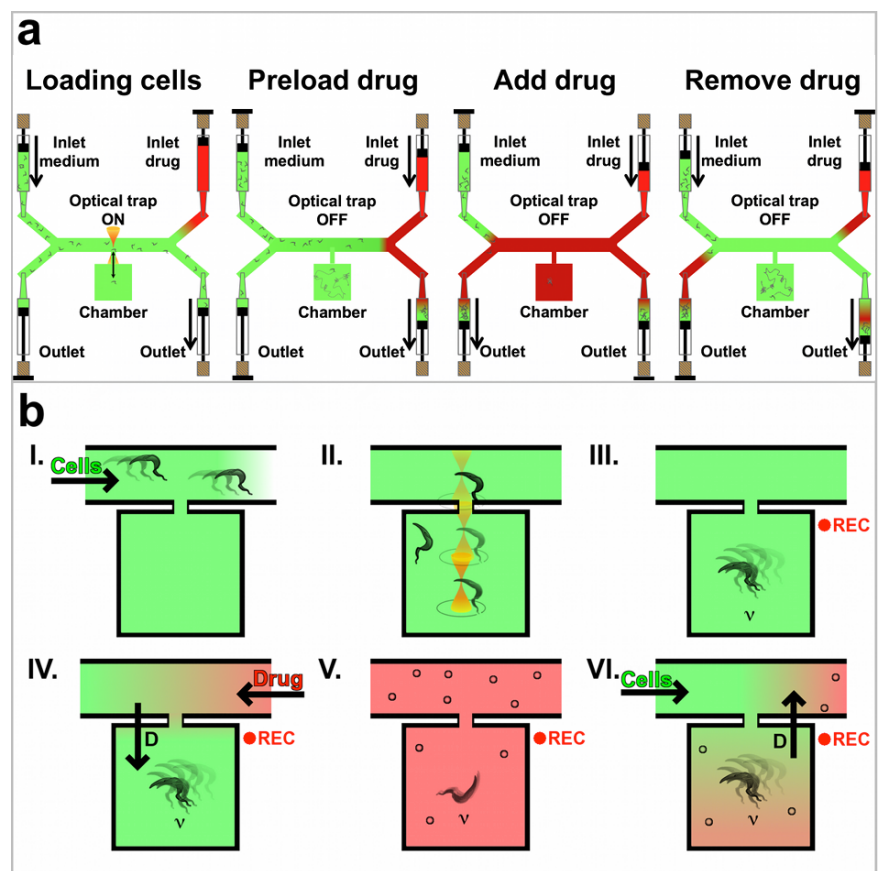

Figure 1: Schematic representation of the device design and experimental procedure. (a): The symmetric device consists of one main channel which supplies square microchambers with media, cells and drugs from the inlets. Excessive liquid is exhausted via the outlets. Inlets and outlets are connected with individually controllable syringe pumps. In combination with the optical trap, cells are loaded into the chambers. Due to the syringe pump control, the drug can be preloaded on the drug side, introduced into the main channel and removed at will. (b): The general procedure consists of two phases. In the loading phase, the device is loaded with cells and medium (I), cells are placed inside the microchambers using optical tweezers (II) and their displacement is recorded (III). During the drug phase, drug-solution is pumped through the main channel and diffuses into the microchambers (IV) until equilibrium is reached (V). Eventually, drug free cell medium is pumped through the main channel and the drug concentration inside the microchambers decreases. During the entire drug phase, cell displacement is recorded.
The dimensions of the connecting channels shield the chambers from the high flow velocities of the main channel and thus, only diffusion-driven material transport into the chambers occurs (supporting information $\mathrm{S} 1$ ). ${ }^{37}$

All inlets and outlets are attached to controlled microliter syringe pumps via Teflon tubing. The syringe pumps can be controlled individually, while the device rests on the stage of a microscope.

\section{Device operations}

At the beginning of an experiment, the device is flushed with culture medium and cells from the cell inlet (step I). When all chambers are filled with medium, the flow is stopped. Using optical tweezers, individual trypanosomes are trapped, moved through the connecting channel into the microchambers and then released (step II, see also supplementary video S3). ${ }^{10}$ Once the chosen number of cells occupies the chambers, cell motility is recorded in a series of images at about 10 frames per second (step III). With the used chamber size (e.g. $75 \mu \mathrm{m} \times 75 \mu \mathrm{m}$ ) the chosen number reflects the maximum number of cells that can be recorded simultaneously without an interference of their trajectories (e.g. 2-4). Subsequently, the drug solution is pumped from the drug inlet through the main channel (step IV). To trace the flow of incoming drug solution, polystyrene spheres $(1 \mu \mathrm{m}$ diameter $)$ are added to the drug solution. While the drug diffuses into the microchambers, a steady flow in the main channel is maintained at $\mathrm{v}_{\text {main }} \approx 2 \mathrm{~mm} / \mathrm{s}$. Image recording is continued until after the drug concentrations inside the chambers and the main channel reached (step V) or, if applicable, until the drug is completely washed out by pumping drug-free culture medium through the device (step VI).

\section{Glutaraldehyde motility assay}

We tested the feasibility of single cell analysis in microfluidic devices by exposing confined trypanosomes to GA from the drug inlet following the procedure described above. The effects of GA on trypanosome motility were recorded with a frame rate of $10 \mathrm{~Hz}$ before and during the inflow of GA.

Based on sequential images, we determined the centre of mass trajectories of the trypanosomes (figure 2) and calculated their velocity, $v(t)=\frac{\sqrt{(r(t+\Delta t)-r(t))^{2}}}{\Delta t}$, where $r$ is the position, $t$ is the time and $\Delta t$ is the interval between two consecutive images. Furthermore, the mean square displacement of the trajectories, $\mathrm{MSD} \equiv\left\langle r(\tau)^{2}\right\rangle=\left\langle(r(t+\tau)-r(t))^{2}\right\rangle$, where $\tau$ is the time interval, was computed. MSD is a common measure for cellular motion $^{10}$ and can be interpreted as the space which is covered over time. In two dimensions, the time dependence of the MSD can be described by the following equation: $\left\langle r(\tau)^{2}\right\rangle=4 \cdot M_{\alpha}$. $\tau^{\alpha}$, where $\alpha$ is the scaling exponent and $M_{\alpha}$ the motility coefficient. ${ }^{40}$ For $\alpha>1$, the motion is super-diffusive, for $\alpha<1$ sub-diffusive and for $\alpha=1$ diffusive or random walk-like. ${ }^{40,41}$ 
Figure 2 shows the color-coded time-lapse trajectories of two confined trypanosomes over six minutes in culture medium (2a,b) and after GA inflow (2c; see also supplementary video S4). At the onset of the experiment, when the device is filled with culture medium, trypanosomes in microchambers were highly motile (figure 2b). Exposure to GA led to a rapid fixation of trypanosomes (figure 2c). Velocity analyses revealed that their initial velocity of about $3.5 \mu \mathrm{m} / \mathrm{s}$ (green) decreased within 1 second to almost zero (figure $2 \mathrm{~d}$,e and supplementary video S4) after contact with GA (magenta). The MSD of trypanosomes moving in a microchamber for $500 \mathrm{~s}$ prior to fixation (green) and after fixation (magenta) are shown in figure $2 \mathrm{f}$. The MSD of motile cells show that trypanosome motility is random walk like. At much longer time scales, the confinement by the microchamber walls drives the MSD into a plateau.

Conversely, GA-fixed trypanosomes exhibited Brownian rather than self-propelled motion. This difference results in a downshifting of the MSD of fixed cells by about two orders of magnitude. As expected, the fixation process was irreversible, since the trypanosomes did not regain motility after the GA drug solution in the device had been replaced by culture medium (figure $2 \mathrm{c}$ ).

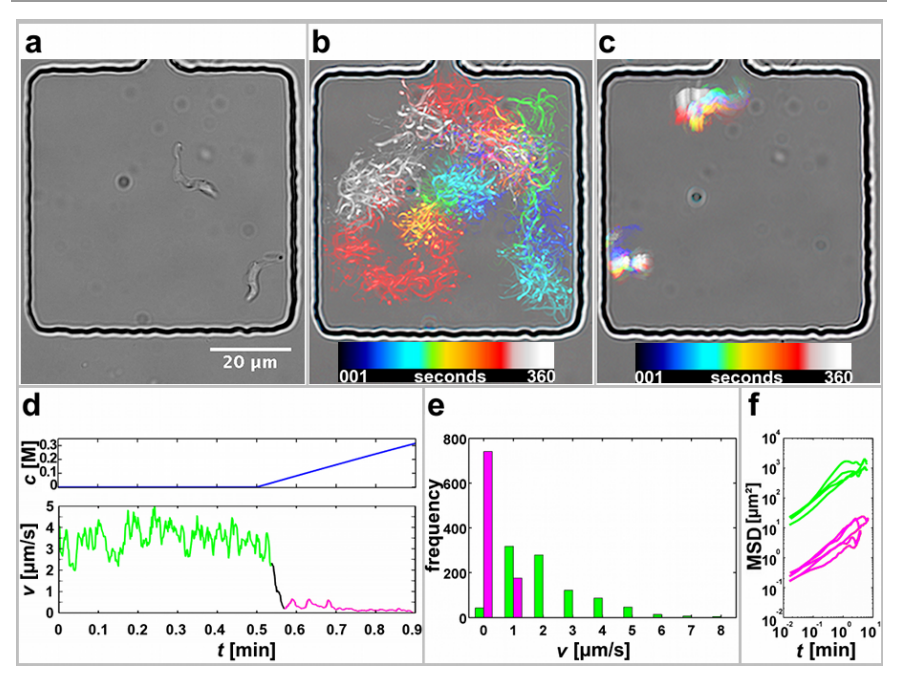

Figure 2: In situ cell fixation. (a) Bright-field micrograph of two trypanosomes confined to a microchamber. (b) Overlaid color-coded time-lapse trajectories of trypanosomes over $6 \mathrm{~min}$ in culture medium. (c) Time-lapse trajectories over 6 min after the addition of GA. (d) Velocity vs. time plot of a trypanosome before (green), during (black) and after (magenta) fixation with GA. (e) Velocity histogram of several trypanosomes before (green) and after (magenta) fixation with GA. (f) MSD of several trypanosomes in confinement before (green) and after GA fixation (magenta).

\section{2-Deoxy-D-glucose (2DG) motility assay}

$2 \mathrm{DG}$ is a glucose analogue that has previously been used to investigate trypanosome metabolism and motility..$^{10,20,42,43}$ For our experiments, trypanosomes have been loaded into the medium-filled microchambers by optical tweezers and then the drug (i.e. 2DG) was preloaded and added at concentrations ranging from $4 \mathrm{mM}$ to $1.05 \mathrm{M}$ (figure 1). 2DG diffused into the microchambers until it reached the concentration as in the main channel (supplementary video S1 and figure S1). Figure 3a illustrates how the concentration of 2DG increased gradually for 5 minutes and then was reduced again to zero along with the concurrent changes of trypanosome swimming velocity $v(t)$. Trypanosome motility gradually decreased with the inflow of 2DG. After approximately 4 minutes of 2DG inflow, motility suddenly ended. Correspondingly, beyond this critical concentration, $v(t)$ was virtually zero (supplementary video S5). However, if the drug was removed by flushing the device with culture medium, trypanosomes started to move again at a nearly constant $v(t)$ (supplementary video S6). It was even possible to repetitively paralyze and re-activate the same trypanosomes. The histograms of $v(t)$ and MSD for several trypanosomes before, during and after paralysis are shown in figure $3 b$ and $3 c$. All MSD exhibit a mean slope of $\alpha \approx 1$. In comparison to motile cells, the MSD of paralyzed cells were downshifted by about two orders of magnitude, thus resembling those of trypanosomes fixed by GA.

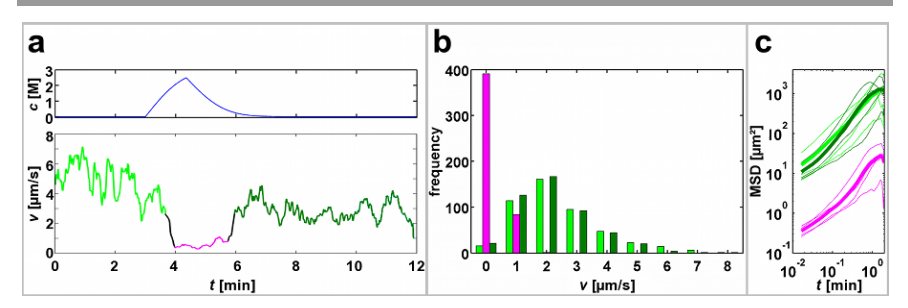

Figure 3: Reversible paralyzing of trypanosomes with 2DG. (a) Concentration of 2DG in the chamber (top) with representative velocity vs. time plot of an individual trypanosome (bottom). At $t=3 \mathrm{~min}$, 2DG started diffusing into the microchamber and the cell velocity decreased (green). After the 2DG concentration reached the critical value (here around $4 \mathrm{~min}$ ), the trypanosome was completely paralyzed (magenta). In response to the inflow of culture medium, 2DG diffused out of the chamber. After approximately $6 \mathrm{~min}$, the concentration of 2DG was below the critical value and the trypanosome regained mobility (dark green). (b) Histogram of the velocities of several trypanosomes before (green) and above the critical value (magenta) of 2DG was reached, as well as after 2DG concentration fell below the critical value (dark green). (c) MSD of trypanosomes at corresponding 2DG concentrations.

From the simulations of the evolution of the 2DG concentrations in the microchambers applied to the corresponding motility analysis, we calculated the concentration of 2DG at which trypanosomes were paralyzed. The percentage of trypanosomes found to be paralyzed at specific concentrations is plotted in figure 4. Full paralysis in trypanosomes occurred at 2DG concentrations of $400 \mathrm{mM}$ and higher. At around $515 \mathrm{mM}, 50 \%$ of the trypanosomes were paralyzed, resulting in the paralyzing dosage, $\mathrm{PD}_{50}=515 \mathrm{mM}$. This paralyzing dosage of $515 \mathrm{mM}$ has been confirmed by experiments with steady concentrations of $2 \mathrm{DG}$.

Moreover, our method allowed for the direct observation of the evolution of the paralyzing effect on trypanosomes. With increasing dosage of 2DG, the trypanosomes moved slower and at concentrations close to $\mathrm{PD}_{50}$, the flagellum was still moving, whereas the cell body was immobilized (supplementary videos S4 \& S5). 


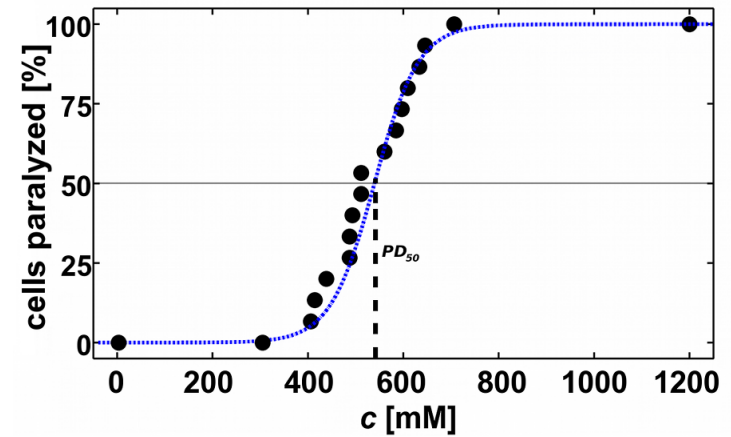

Figure 4: Paralyzing dosage $\mathrm{PD}_{50}$. Percentage of trypanosomes paralyzed vs. concentration of $2 \mathrm{DG}$. $\mathrm{PD}_{50}$ denotes the concentration of $2 \mathrm{DG}$ at which $50 \%$ of trypanosomes are paralyzed.

\section{Motility factor and low dosage assay}

We examined the effects of 2DG on trypanosome motility at concentrations two orders of magnitude lower than the measured $\mathrm{PD}_{50}$. For these low concentrations the recorded videos showed no obvious impact on trypanosome motility. But an increase of 2DG correlated with a downshift of MSD curves even at low concentrations (figure 5a).

For a more quantitative description of the impact of 2DG on the motility of trypanosomes, we used a simple hydrodynamic model. Whereas paralyzed cells exclusively exhibited Brownian motion, the motile cells at different 2DG concentrations were characterized by a random walk. Using the Stokes-Einstein relation, the motility coefficient of paralyzed cells, $M_{\text {para }}$, can be related to the thermal energy, $k_{B} T$. The friction factor of a paralyzed trypanosome, $\xi_{\text {para }}$, is linked to its diffusion coefficient, $\mathrm{D}_{\text {para }}$, by $M_{\text {para }}=\frac{\left\langle r(\tau)^{2}\right\rangle_{\text {para }}}{4 \tau}=D_{\text {para }}=$ $\frac{k_{B} T}{\xi_{\text {para }}}$. By analogy, the motility coefficient of the motile, randomly walking cells $(\alpha=1), M_{\text {motile }} \approx M_{\alpha=1}=\frac{\left\langle r(\tau)^{2}\right\rangle_{\text {motile }}}{4 \tau}$, can be described as $M_{\text {motile }} \cong \frac{\tilde{\epsilon} k_{B} T}{\xi_{\text {motile }}}$, using a virtual temperature, $\tilde{\epsilon} T$, or an apparent motility energy, $\tilde{\epsilon} k_{B} T$, where $\tilde{\epsilon}$ is a dimensionless factor. Assuming that the friction factors of motile and paralyzed cells are almost identical, $\xi_{\text {para }} \approx \xi_{\text {motile }}$, the motility factor, $\tilde{\epsilon}$, can be obtained by $\tilde{\epsilon} \cong \frac{M_{\text {motile }}}{M_{\text {para }}}=$ $\frac{\left\langle r(\tau)^{2}\right\rangle_{\text {motile }}}{\left\langle r(\tau)^{2}\right\rangle_{\text {para }}}$. The motility factor $\tilde{\epsilon}(c)$ as a function of the $2 \mathrm{DG}$ concentration, which is a coarse estimate of the motility energy in multiples of $k_{B} T$, is presented in figure 5b. A motile trypanosome in culture medium utilizes a motility energy that is approximately 130 times greater than thermal energy. The motility factor $\tilde{\epsilon}(c)$ shows a strong, exponential-like decay with increasing concentration of 2DG $(0-400 \mathrm{mM})$. However, for $\tilde{\epsilon}(c) \gg 1$, the trypanosomes were still motile. At 400 to $680 \mathrm{mM}$ 2DG, the trypanosomes became paralyzed with a $\mathrm{PD}_{50}=515 \mathrm{mM}$ (figure 4). For a motility factor $\tilde{\epsilon}(c)=1$ the motility energy of a trypanosome equals the thermal energy, $k_{B} T$. Thus, propulsion is arrested and only Brownian motion occurs.

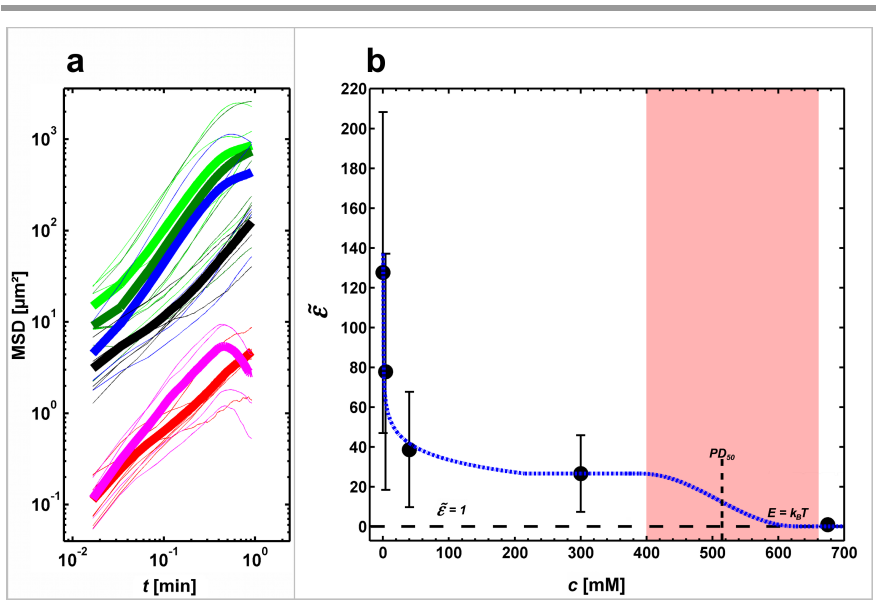

Figure 5: Analysis of the impact of 2DG on trypanosome propulsion. (a) MSD of trypanosomes in identical chambers but at different concentrations of 2DG in the medium (bright green: drug-free culture medium, dark green: $c_{2 \mathrm{DG}}=4 \mathrm{mM}$, blue: $c_{2 D G}=40 \mathrm{mM}$, black: $c_{2 D G}=300 \mathrm{mM}$, magenta: fixed with $\mathrm{GA}$, red: paralyzed; $c_{2 D G}=700 \mathrm{mM}$ ). Thin lines are MSD of individual cells, bold lines are averages of a number of cells at the same 2DG concentration. (b) The motility factor $\tilde{\epsilon}$ plotted against the concentration of 2DG in the medium. The dashed black line represents a motility factor $\tilde{\epsilon}=1$, which means that the propulsion energy of a trypanosome equals the thermal energy, $k_{B} T$. The area shaded in red corresponds to the concentration range shown in figure 4 . Dashed blue line serves as a guide to the eye.

\section{Suramin motility and vitality assay}

Suramin was introduced almost a century ago, and despite its severe side effects, still is the drug of choice in treating early stages of Eastern African sleeping sickness in humans. ${ }^{44-46}$ The route of entry into the trypanosomes as well as the mode of action remain enigmatic. Suramin presumably associates with different serum proteins in the bloodstream, e.g. low-density lipoproteins (LDL). ${ }^{46,47}$ It has been proposed that suramin's trypanocidal activity arises from its interference with endocytic uptake of LDL. ${ }^{47}$ While it has been indicated that suramin might inhibit several enzymes and receptors promiscuously ${ }^{46}$, a more recent study suggests that suranim undergoes receptormediated endocytosis to the lysosome where it escapes to the cytoplasm and may inhibit cellular processes ${ }^{48}$.

To gain a better understanding on the effects of suramin, we tested the response of trypanosomes to the drug in our single cell analysis. Similar to $2 \mathrm{DG}$, suramin induced a paralysis of confined cells at a critical concentration of $\mathrm{PD}_{50}=123 \mathrm{mM}$, (figure 6a). Moreover, when the drug was flushed out of the device by culture medium, paralyzed trypanosomes regained motility.

We quantitatively assessed the impact of suramin on the motility of trypanosomes on the basis of MSDs that largely exhibit a mean slope of $\alpha \approx 1$ (figure 6c). At a concentration of $3.5 \mathrm{mM}$ suramin, which is significantly below $\mathrm{PD}_{50}$, the motility factor $\tilde{\epsilon}$ was reduced to about one third as compared to trypanosomes in a drug-free environment. Between $35 \mathrm{mM}$ and $80 \mathrm{mM}$ suramin, a steady state with $\tilde{\epsilon}(c) \approx 35$ was reached, while between 80 and $170 \mathrm{mM}$, paralysis resulted in $\tilde{\epsilon}(c)$ of 1 (figure 6d). 
Besides the reversible reduction in cell motility, suramin exhibited trypanocidal effects. At dosages above $35 \mathrm{mM}$, trypanosomes were found to start rupturing and disintegrating after about 6 minutes of exposure to suramin (supplementary video S7). The percentage of trypanosomes that disintegrated at the end of a time lapse of $14 \mathrm{~min}$ increases with the concentration of suramin at steady state (figure 6b). Interestingly, the suramin-triggered disintegration occurred even after suramin removal, indicating that suramin either accumulates in trypanosomes or irreversibly alters cell regulations.
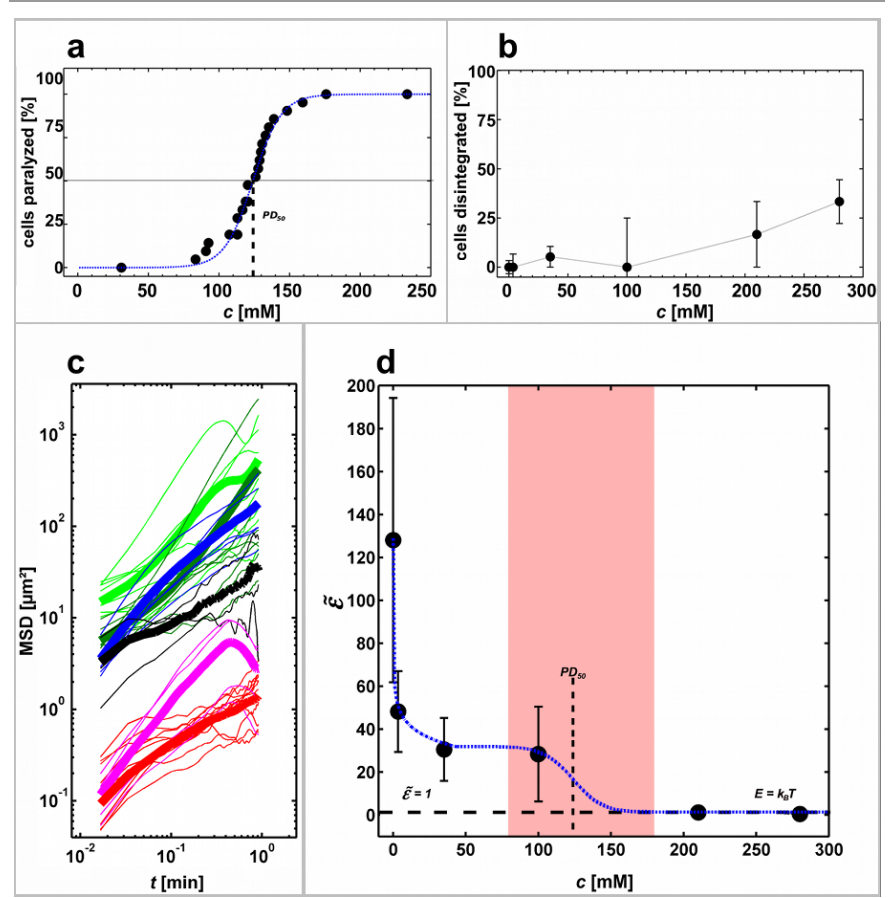

Figure 6: Quantitative analysis of the impact of suramin on trypanosome propulsion and disintegration. (a) Concentration dependent paralysis by suramin. $\mathrm{PD}_{50}$ denotes the concentration of suramin at which $50 \%$ of trypanosomes are paralyzed $(123 \mathrm{mM})$. (b) Disintegration of trypanosomes in response to different suramin concentrations. (c) MSD of confined trypanosomes over time (bright green: drug free medium, dark green: $c_{\text {suramin }}=3.5 \mathrm{mM}$, blue: $c_{\text {suramin }}=35 \mathrm{mM}$, black: $c_{2 \mathrm{DG}}=100 \mathrm{mM}$, magenta: fixed with GA, red: paralyzed; $c_{\text {suramin }}=210 \mathrm{mM}-$ $280 \mathrm{mM}$ ). Thin lines represent MSD of individual cells, bold lines are averages at the respective suramin concentrations. (d) The motility factor $\tilde{\epsilon}$ at different concentrations of suramin in the microchamber. The dashed black line represents $\tilde{\epsilon}=1$, which indicates that the propulsion energy of a trypanosome equals the thermal energy, $k_{B} T$. The red area represents the concentration range shown specified in figure 6a. Dashed blue line serves as a guide to the eye.

\section{Conclusions}

The quantitative assessment of reversible and irreversible paralysis of trypanosomes shows that microfluidics in combination with optical micromanipulation is a versatile tool for single cell analysis and pharmaceutical testing. Our straightforward microfluidics-based method allows not only for an in situ analysis of single cells in defined, gradually changeable environments but also for a rapid evaluation of the impact of drugs and chemicals by analysing cell motility. Moreover, this approach eliminates the error of concentration inhomogeneity that arises from adding drug solutions drop-wise into a cell-laden well that can lead to false results in commonly used micro-assays.

The rapid fixation of trypanosomes with GA represents an excellent model for a highly cytocidal drug. We found that exposure to GA immediately and irreversibly ended active motion. This is most likely a result of cross-linking proteins involved in flagellum-dependent parasite motility, e.g. dynein $^{49}$. In contrast, paralysis of trypanosomes was reversible when trypanosomes were exposed to respective concentrations of $2 \mathrm{DG}$, which is a competitive inhibitor of glycolysis ${ }^{43}$. Based on the precise and rapid control of drug concentrations in the confinements, we were able to determine the dosage at which $50 \%$ of the trypanosomes are paralyzed $\left(\mathrm{PD}_{50}\right)$. Furthermore, our data reveal an impact of $2 \mathrm{DG}$ on the motility of the cells at doses that are significantly lower than $\mathrm{PD}_{50}$.

We introduced a simple random walk model to derive the propulsion energy from cell trajectories. According to this model, we noted a reduction of propulsion energy with increasing concentration of $2 \mathrm{DG}$. This finding is consistent with the decrease in cell motility and with reports of 2DG as inhibitor of glycolysis in Trypanosoma brucei bloodstream forms. ${ }^{43}$ It is conceivable that the resulting depletion of ATP might cause a decrease in flagellar motor activity and thereby reduce cell motility and hence vitality.

As the cytolysis-free, microfluidics-based single cell analysis revealed effects of $2 \mathrm{DG}$ at concentrations significantly lower than those used in common drug testing systems and had a much faster assessment than regularly used assays, ${ }^{4,6}$ we examined the response of trypanosomes to suramin, which is still the most commonly used drug in early-stage trypanosomiasis. Our data show that suramin and 2DG have comparable effects with respect to cell motility, which is likely due to suramin blocking endocytosis in trypanosomes. Unlike 2DG, suramin also caused individual trypanosomes to rupture and disintegrate after exposure to concentrations of $35 \mathrm{mM}$ and higher. The cytocidal effect of suramin suggests that the two drugs affect different cellular mechanisms that ultimately result in the inhibition of motility.

While we characterized our microfluidics-based method with established drugs and chemicals that affect Trypanosoma brucei brucei, the system lends itself to numerous adaptations, like finding and characterizing new drugs against trypanosomes or any other pathogenic cell. Further versatility can be easily accomplished by integrating additional methodologies, such as screening for mutants or fluorescence imaging - live as well as after fixation. Moreover, by applying our device to human cells and to pathogens simultaneously, effective drug doses could be determined in a single experiment.

\section{Acknowledgements}

We thank Cora-Ann Schoenenberger for critical reading of the manuscript and the Swiss Tropical Public Health Institute for 
the gift of the Trypanosoma brucei brucei MiTat 1.2. A. H. wants to thank Stefanie J. Krauth for fruitful discussions. We gratefully acknowledge funding from the DFG within the priority program SPP1207 (EN305/4, PF375/5) and from the SNF 200020_141270.

\section{Notes and references}

${ }^{a}$ Department of Chemistry, University of Basel, 4056 Basel, Switzerland.

${ }^{b}$ Department of Complex Fluids, Max Planck Institute for Dynamics and Self-Organization, 37073 Göttingen, Germany.

${ }^{c}$ Department of Cell and Developmental Biology, Biocentre, University of Würzburg, 97074 Würzburg, Germany.

${ }^{1}$ present address: DESY Photon Science, 22607 Hamburg, Germany

2 present address: Department of Bionanoscience, Delft University of Technology, Lorentzweg 1, 2628 CJ Delft, The Netherlands

${ }^{3}$ present address: Department of Chemical and Biological Engineering,

Princeton University, Princeton, NJ 08544, USA

1. X. T. Zheng, L. Yu, P. Li, H. Dong, Y. Wang, Y. Liu, and C. M. Li, Adv. Drug Deliv. Rev., 2013, 65, 1556-74.

2. H. Andersson and A. van den Berg, Sensors Actuators B Chem., 2003, 92, 315-325.

3. P. Neuži, S. Giselbrecht, K. Länge, T. J. Huang, and A. Manz, Nat. Rev. Drug Discov., 2012, 11, 620-32.

4. A. M. C. Canavaci, J. M. Bustamante, A. M. Padilla, C. M. Perez Brandan, L. J. Simpson, D. Xu, C. L. Boehlke, and R. L. Tarleton, PLoS Negl. Trop. Dis., 2010, 4, e87:0907-13.

5. N. Van Reet, P. Pyana, S. Rogé, F. Claes, and P. Büscher, Parasit. Vectors, 2013, 6, 207.

6. M. De Rycker, S. O’Neill, D. Joshi, L. Campbell, D. W. Gray, and A. H. Fairlamb, PLoS Negl. Trop. Dis., 2012, 6, e1932:1-7.

7. H. Yin and D. Marshall, Curr. Opin. Biotechnol., 2012, 23, 110-9.

8. E. Brouzes, M. Medkova, N. Savenelli, D. Marran, M. Twardowski, J. B. Hutchison, J. M. Rothberg, D. R. Link, N. Perrimon, and M. L. Samuels, Proc. Natl. Acad. Sci. U. S. A., 2009, 106, 14195-200.

9. M. Junkin and S. Tay, Lab Chip, 2014, 14, 1246-60.

10. M. Engstler, N. Heddergott, T. Krüger, E. Stellamanns, S. Uppaluri, and T. Pfohl, in Nature-inspired Fluid Mechanics, eds. C. Tropea and H. Bleckmann, Heidelberg, Springer-V., 2012, pp. $43-61$.

11. H. Zhang and K.-K. Liu, J. R. Soc. Interface, 2008, 5, 671-90.

12. M. M. Wang, E. Tu, D. E. Raymond, J. M. Yang, H. Zhang, N. Hagen, B. Dees, E. M. Mercer, A. H. Forster, I. Kariv, P. J.

Marchand, and W. F. Butler, Nat. Biotechnol., 2005, 23, 83-7.

13. T. L. Min, P. J. Mears, L. M. Chubiz, C. V Rao, I. Golding, and Y. R. Chemla, Nat. Methods, 2009, 6, 831-5.
14. A. Groisman, C. Lobo, H. Cho, J. K. Campbell, Y. S. Dufour, A. M. Stevens, and A. Levchenko, Nat. Methods, 2005, 2, 685-689.

15. C.-C. Hsieh, S.-B. Huang, P.-C. Wu, D.-B. Shieh, and G.-B. Lee, Biomed. Microdevices, 2009, 11, 903-13.

16. D. Ryan, K. Ren, and H. Wu, Biomicrofluidics, 2011, 5, 21501-121501-9.

17. D. Wang and S. Bodovitz, Trends Biotechnol., 2010, 28, 281-90.

18. F. Asphahani, K. Wang, M. Thein, O. Veiseh, S. Yung, J. Xu, and M. Zhang, Phys. Biol., 2011, 8, 015006.

19. A. T. Ohta, M. Garcia, J. K. Valley, L. Banie, H.-Y. Hsu, A Jamshidi, S. L. Neale, T. Lue, and M. C. Wu, Lab Chip, 2010, 10, 3213-3217.

J. R. Seed, M. A. Baquero, and J. F. Duda, Exp. Parasitol., 1965, 16, 363-368.

21.

K. Fenn and K. R. Matthews, Curr. Opin. Microbiol., 2007, 10, 539-46.

K. R. Matthews, J. R. Ellis, and A. Paterou, Trends Parasitol., 2004, 20, 40-47.

N. Rusk, Nat. Methods, 2005, 2, 572-572.

R. Brun, J. Blum, F. Chappuis, and C. Burri, Lancet, 2010, 375, 148-59.

S. Welburn, K. Picozzi, E. Fevre, and P. Coleman, Lancet, 2001, 358, 2017-2019.

E. M. Fèvre, P. G. Coleman, M. Odiit, J. W. Magona, S. C. Welburn, and M. E. Woolhouse, Lancet, 2001, 358, 625-8.

28. K. Picozzi, E. M. Fèvre, M. Odiit, M. Carrington, M. C. Eisler, I. Maudlin, and S. C. Welburn, BMJ, 2005, 331, 1238-41.

29. S. Welburn, E. Fèvre, and P. Coleman, Trends Parasitol., 2001, 17, 19-24.

30. S. C. Welburn, P. G. Coleman, I. Maudlin, E. M. Fèvre, M. Odiit, and M. C. Eisler, Trends Parasitol., 2006, 22, 123-8.

31. P. Overath and M. Engstler, Mol. Microbiol., 2004, 53, 735-44.

32. M. Engstler, T. Pfohl, S. Herminghaus, M. Boshart, G. Wiegertjes, N. Heddergott, and P. Overath, Cell, 2007, 131, 505-515.

33. A. Y. Koyfman, M. F. Schmid, L. Gheiratmand, C. J. Fu, H. a Khant, D. Huang, C. Y. He, and W. Chiu, Proc. Natl. Acad. Sci. U. S. A., 2011, 108, 11105-8. 
34. Z. B. Mackey, A. M. Baca, J. P. Mallari, B. Apsel, A. Shelat, E. J. Hansell, P. K. Chiang, B. Wolff, K. R. Guy, J. Williams, and J. H. McKerrow, Chem. Biol. Drug Des., 2006, 67, 355-63.

35. E. Myburgh, J. a Coles, R. Ritchie, P. G. E. Kennedy, A. P. McLatchie, J. Rodgers, M. C. Taylor, M. P. Barrett, J. M. Brewer, and J. C. Mottram, PLoS Negl. Trop. Dis., 2013, 7, e2384:1-12.

36. H. Hirumi and K. Hirumi, 2014, 75, 985-989.

37. S. Deshpande and T. Pfohl, Biomicrofluidics, 2012, 6, 34120.

38. P. Th, U. E. Ruttimann, and M. Unser, IEEE Trans. Image Process., 1998, 7, 27-41.

39. J. Schindelin, I. Arganda-Carreras, E. Frise, V. Kaynig, M. Longair, T. Pietzsch, S. Preibisch, C. Rueden, S. Saalfeld, B. Schmid, J.-Y. Tinevez, D. J. White, V. Hartenstein, K. Eliceiri, P. Tomancak, and A. Cardona, Nat. Methods, 2012, 9, 676-82.

40. S. Uppaluri, J. Nagler, E. Stellamanns, N. Heddergott, S. Herminghaus, M. Engstler, and T. Pfohl, PLoS Comput. Biol., 2011, 7, e1002058:1-8.

41. Y. Han, a M. Alsayed, M. Nobili, J. Zhang, T. C. Lubensky, and a G. Yodh, Science, 2006, 314, 626-30.

42. N. Lamour, L. Rivière, V. Coustou, G. H. Coombs, M. P. Barrett, and F. Bringaud, J. Biol. Chem., 2005, 280, 11902-10.

43. M. Parsons and B. Nielsen, Mol. Biochem. Parasitol., 1990, 42, 197-203.

44. D. Legros, G. Ollivier, M. Gastellu-etchegorry, C. Paquet, C. Burri, J. Jannin, and P. Büscher, Lancet, 2002, 2, 437-440.

45. A. J. Nok, Parasitol. Res., 2003, 90, 71-9.

46. A. H. Fairlamb, Trends Parasitol., 2003, 19, 488-494.

47. E. L. M. Vansterkenburg, I. Coppens, J. Wilting, O. J. M. Bos, M. J. E. Fischer, L. H. M. Janssen, and F. R. Opperdoes, Acta Trop., 1993, 54, 237-250.

48. S. Alsford, M. C. Field, and D. Horn, Trends Parasitol., 2013, 29, 207-12.

49. L. C. Hughes, K. S. Ralston, K. L. Hill, and Z. H. Zhou, PLoS One, 2012, 7, e25700:1-10. 